\title{
Epitaxial nanocrystalline tin dioxide thin films grown on (0001) sapphire by femtosecond pulsed laser deposition
}

\author{
J. E. Dominguez, L. Fu, and X. Q. Pan ${ }^{\text {a) }}$ \\ Department of Materials Science and Engineering, The University of Michigan, Ann Arbor, Michigan 48109
}

(Received 12 April 2001; accepted for publication 25 May 2001)

\begin{abstract}
Nanocrystalline tin dioxide $\left(\mathrm{SnO}_{2}\right)$ thin films of different thicknesses were fabricated on the (0001) surface of $\alpha-\mathrm{Al}_{2} \mathrm{O}_{3}$ (sapphire) using femtosecond pulsed laser deposition. X-ray diffraction and transmission electron microscopy (TEM) analysis revealed that the microstructure of the films strongly depends on the film thickness. The films with a small thickness $(<30 \mathrm{~nm})$ are composed of nanosized columnar (100) oriented grains $(3-5 \mathrm{~nm}$ in diameter) which grow epitaxially on the substrate with three different in-plane grain orientations. The (101) oriented grains $(25 \mathrm{~nm}$ in diameter) appear when the film thickness becomes larger than a critical value (about $60 \mathrm{~nm}$ ). The volume fraction of the (101) grains increases with film thickness. Cross-section TEM studies indicated that the (101) oriented grains nucleate on the top of the (100) oriented nanosized grains and show abnormal grain growth driven by surface energy minimization. As a result, the electrical transport properties are strongly dependent on the film thickness. (C) 2001 American Institute of Physics. [DOI: 10.1063/1.1386406]
\end{abstract}

The unique materials properties found on the nanometer scale and the miniaturization of modern semiconductor devices have prompted the widespread investigation of the properties of nanocrystalline materials. ${ }^{1,2}$ On this scale, the behavior of functional materials is strongly size dependent and interface controlled. $\mathrm{SnO}_{2}$ with the rutile structure is a wide energy gap $(3.6 \mathrm{eV}) n$-type semiconductor. ${ }^{3}$ Owing to its outstanding electrical, optical, and electrochemical properties, $\mathrm{SnO}_{2}$ is extensively used in many applications such as catalytic support material, transparent electrodes for flat panel displays and solar cells, ${ }^{4}$ and gas sensors. ${ }^{5}$ In particular, $\mathrm{SnO}_{2}$ thin films have drawn much interest because of their potential application in microsensor devices. ${ }^{6}$ Considerable attention has recently focused on the development of solid-state gas sensors based on thin films with a crystallite size smaller than the Debye length of the material, which show an increased gas sensitivity and short response time. ${ }^{7}$ Taking into account its potential application and the importance for fundamental research as well as its simple structure, $\mathrm{SnO}_{2}$ is an ideal model system for a systematic investigation on microstructure, grain boundary and interface characteristics, and their effects on physical properties.

We have fabricated nanocrystalline $\mathrm{SnO}_{2}$ thin films using electron beam deposition followed by postannealing. ${ }^{8-10}$ It was found that the electrical transport and chemical sensing properties of the films strongly depend on the size, orientation, and shape of $\mathrm{SnO}_{2}$ grains in the films. ${ }^{11}$ To understand the structure-property relationship of $\mathrm{SnO}_{2}$ thin films, one needs to fabricate thin films with controlled stoichiometry and microstructures. We have recently synthesized $\mathrm{SnO}_{2}$ thin films with different microstructures using femtosecond pulsed laser deposition (PLD). It has been found that the films deposited on (1012) sapphire substrates at $700{ }^{\circ} \mathrm{C}$ are epitaxial, single crystal, ${ }^{11}$ while those grown on (0001) sap-

a)Electronic mail: panx@umich.edu phire substrates with the otherwise same conditions consist of nanocrystalline grains. In this letter we present our studies on these nanocrystalline $\mathrm{SnO}_{2}$ thin films.

Tin dioxide films with the thickness of 15-100 nm were deposited on (0001) sapphire substrates using femtosecond pulsed laser deposition, under the same conditions as reported elsewhere. ${ }^{11}$ Thin films were deposited at $700{ }^{\circ} \mathrm{C}$ with the oxygen backfill pressure of 0.8 mTorr. The microstructure of the films was characterized by x-ray diffraction and transmission electron microscopy (TEM). Electrical conductivity and Hall effect measurements were conducted using a four-point probe and a magnetic field of about $2.2 \mathrm{kG}$, in a pure $\mathrm{N}_{2}$ atmosphere to avoid interference with chemisorbed oxygen.

Figure 1(a) shows the x-ray diffraction pattern of $\mathrm{SnO}_{2}$ films grown on the (0001) sapphire substrates with different thicknesses. It can be seen that the films with a thickness less than $30 \mathrm{~nm}$ show only the (100) orientation, whereas the thicker films $(>30 \mathrm{~nm})$ show both (100) and (101) orientations. Figure $1(b)$ shows the $\{110\}$ pole figure of the $100-n m-$ thick film. Three sets $(\{1,4\},\{2,5\}$, and $\{3,6\})$ of two peaks with high intensities, which are about $45^{\circ}$ away from the center, correspond to the $\{110\}$ poles in the standard (100) projection of $\mathrm{SnO}_{2}$. These three sets of two poles are rotated by $120^{\circ}$ with respect to each other in the azimuthal direction. Another three sets, $\{\mathrm{a}, \mathrm{b}\},\{\mathrm{c}, \mathrm{d}\}$, and $\{\mathrm{e}, \mathrm{f}\}$ which are about $70^{\circ}$ away from the center, correspond to the $\{110\}$ pole of (101) oriented $\mathrm{SnO}_{2}$ grains. These results indicate that three different in-plane orientations are present in both (100) and (101) oriented grains. This is due to the threefold symmetry in the pseudo-hexagonal structure of the (0001) sapphire substrate, which is not present in the (100) and (101) planes of the tetragonal rutile structure. By comparing the pole figure of the film with that of the corresponding substrate, the in-plane orientation relationships of $\mathrm{SnO}_{2}$ grains of both types are determined: $\mathrm{SnO}_{2}(100)[010] \| \mathrm{Al}_{2} \mathrm{O}_{3}(0001)[1 \overline{2} 10]$, $\mathrm{SnO}_{2}(101)[010] \| \mathrm{Al}_{2} \mathrm{O}_{3}(0001)[1 \overline{2} 10]$, and their variants by 

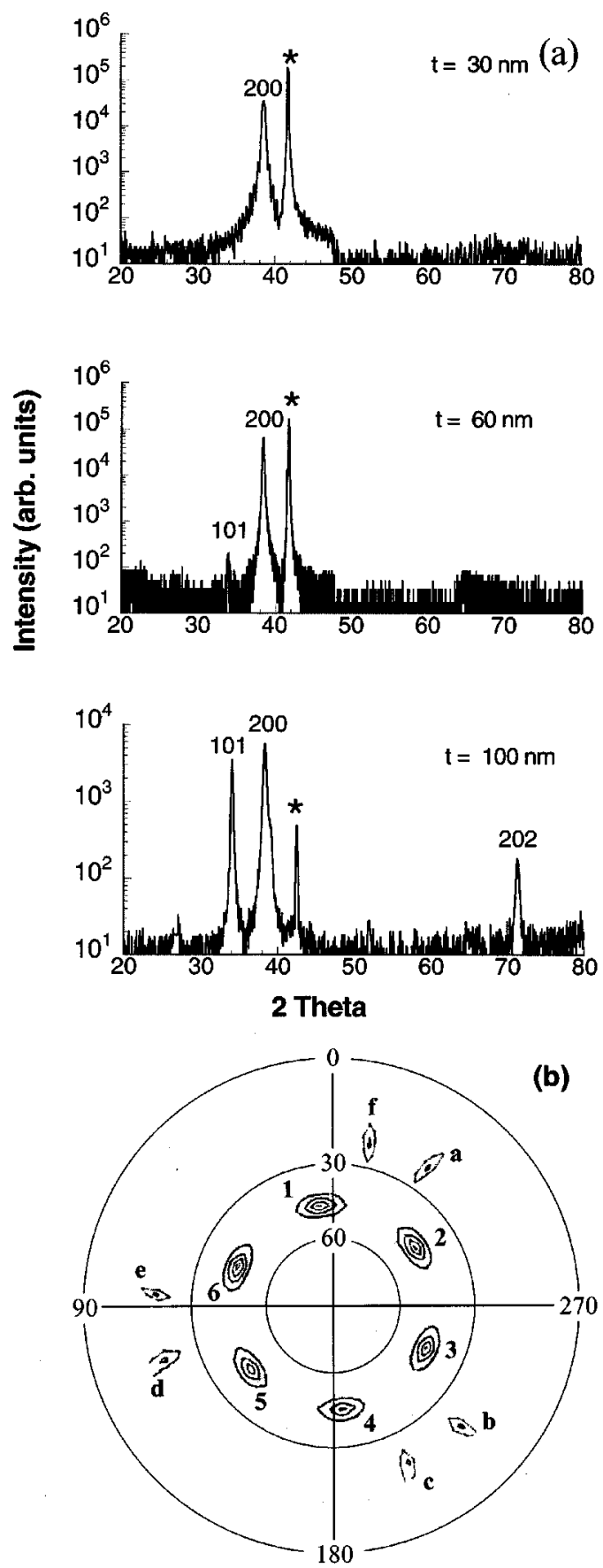

FIG. 1. (a) X-ray diffraction patterns of $\mathrm{SnO}_{2}$ films with different thicknesses. The peaks marked with an asterisk correspond to the substrate (0006) reflection. (b) $\{110\}$ Pole figure of the 100-nm-thick $\mathrm{SnO}_{2}$ film.

a rotation of $\pm 120^{\circ}$ around the film normal, i.e., the [0001] direction of the $\mathrm{Al}_{2} \mathrm{O}_{3}$ substrate.

Figure 2(a) shows the plan view TEM image of a 100$\mathrm{nm}$-thick film. The corresponding electron diffraction pattern is shown as inset, which is the mixture of diffraction spots from both (100) and (101) oriented grains. Selected area electron diffraction and high-resolution transmission electron microscopy (HRTEM) studies revealed that the bright regions in Fig. 2(a) correspond to the (101) grains, while the dark matrix consists of fine (100) oriented grains. Grains of each type consist of three different orientations, coincident with the previous x-ray diffraction studies. The (101) grains have a mean size (diameter) of $25 \mathrm{~nm}$, randomly distributed in the film. Figure 2(b) is a HRTEM image taken from the
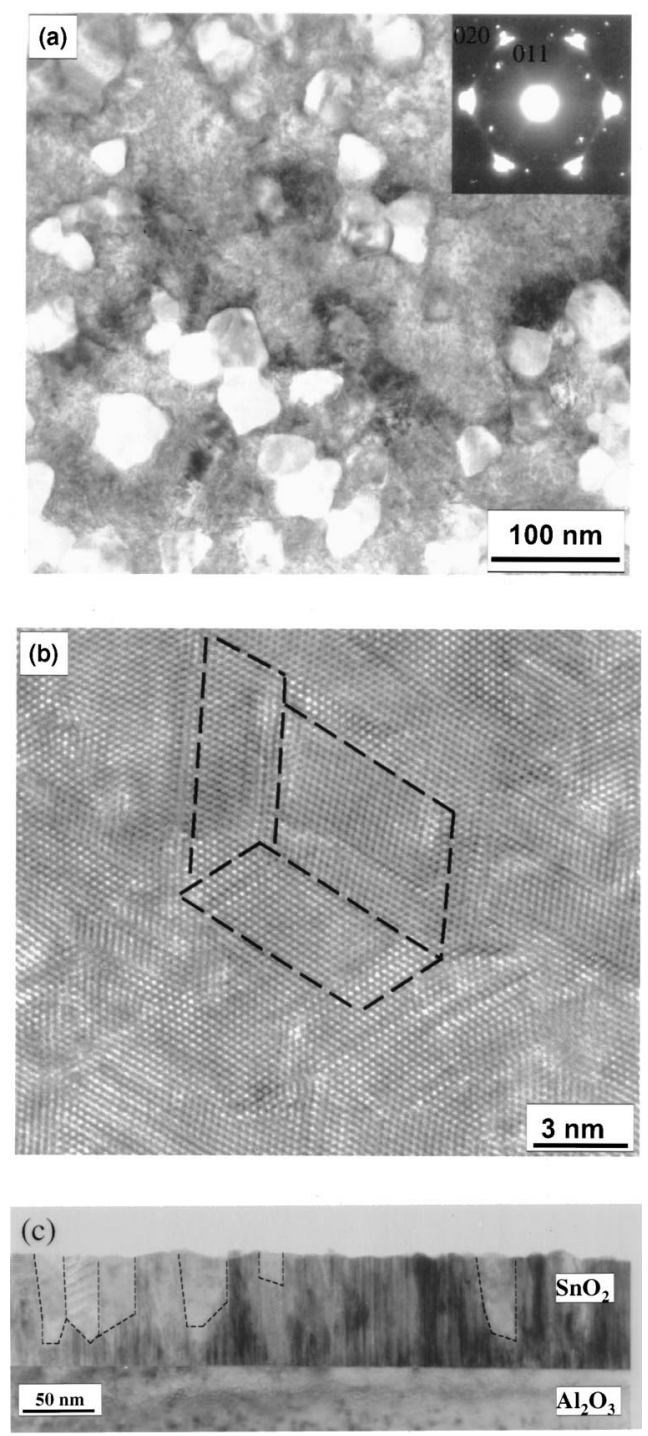

FIG. 2. (a) Plan view TEM image of a 100 -nm-thick $\mathrm{SnO}_{2}$ film grown on (0001) sapphire substrate, showing the existence of both (100) and (101) oriented grains. (b) HRTEM image taken from the dark region in (a) showing nanosized (100) oriented grains. (c) Cross-section TEM image of the same film showing the formation of the (101) grains on top of the (100) nanosized grains.

dark regions in Fig. 2(a), showing fine (100) oriented grains with three different in-plane orientations. The mean diameter of the (100) grains is about $5 \mathrm{~nm}$. Figure 2(c) is a crosssection TEM image of the 100-nm-thick film, showing the columnar shape of both the fine (100) grains and the larger (101) grains outlined by dashed lines. It can also be seen that only the (100) oriented grains exist near the substrate/film interface and the (101) oriented grains occur at a certain distance from the interface. Furthermore, the cross-section HRTEM images of thinner films $(<30 \mathrm{~nm})$ only show (100) oriented grains, which agrees with the x-ray diffraction studies in Fig. 1(a). This reveals that the fine (100) grains are structurally favorable to form on the (0001) sapphire surface, while the (101) oriented grains are kinetically favorable when the film thickness becomes greater than a critical thickness $(\sim 60 \mathrm{~nm})$. It was also found that the mean diameter of (101) oriented grains increases with film thickness. This means that (101) grains grow at the expense of (100) grains as the film grows thicker. 


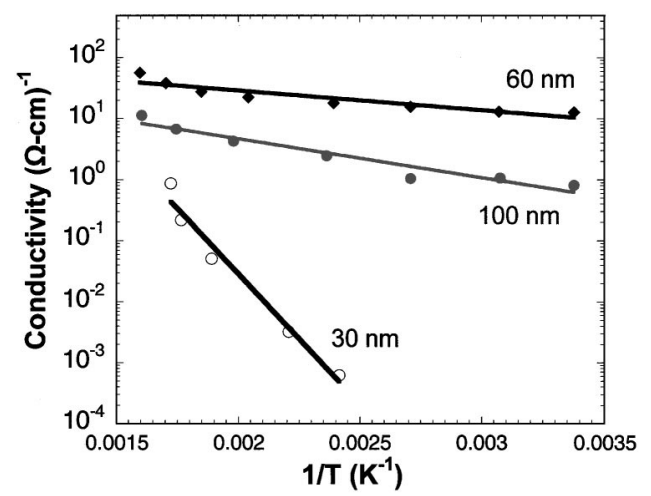

FIG. 3. Electrical conductivity of the $\mathrm{SnO}_{2}$ films as a function of inverse temperature.

The epitaxial growth of (100) oriented grains on the (0001) $\mathrm{Al}_{2} \mathrm{O}_{3}$ substrate is favorable because of: (1) the similar oxygen octahedral networks existing on the $\mathrm{SnO}_{2}(100)$ surface and the $\mathrm{Al}_{2} \mathrm{O}_{3}$ (0001) surface and (2) small lattice mismatch $(<1 \%)$ along the [010] direction which lies along one of the $[1 \overline{2} 10],[\overline{2} 110],[11 \overline{2} 0]$ directions of $\mathrm{Al}_{2} \mathrm{O}_{3}$. The small grain size of the (100) oriented grains may result from the large lattice mismatch (13.8\%) with the substrate along the [001] direction of $\mathrm{SnO}_{2}$. The formation of (101) oriented $\mathrm{SnO}_{2}$ on the (0001) $\mathrm{Al}_{2} \mathrm{O}_{3}$ is not favorable because there is no good lattice match between the $\mathrm{SnO}_{2}$ (101) surface and the $\mathrm{Al}_{2} \mathrm{O}_{3}$ (0001) surface. The occurrence of (101) grains in the late stage of film growth is probably the product of surface energy minimization. ${ }^{12}$ They form on the (100) grains, which have three orientations in the film plane, resulting in three different in-plane orientations of the (101) grains.

Figure 3 shows the conductivity of the $\mathrm{SnO}_{2}$ films with a thickness ranging from 30 to $100 \mathrm{~nm}$. The 30-nm-thick film shows the lowest conductivity and the 60 -nm-thick film has the highest value. The electron concentration and Hall mobility as a function of inverse temperature for all the films were also obtained, showing the same trend observed for the conductivity of the films.

The exponential behavior of the transport properties is consistent with a grain boundary electrical barrier model in polycrystalline films. ${ }^{13}$ A greater barrier potential indicates a larger amount of charge trapped at the grain boundaries or a smaller number of free electrons in the film. The 30-nm-thick film shows the lowest electron concentration due to its high density of grain boundaries that can trap a larger number of electrons. Furthermore, the trapped electrons at the grain boundary will build up a potential barrier for electrical conduction, reducing the mobility of conduction electrons. With increasing film thickness, the mean grain diameter increases in order to lower the grain boundary energy in the film. The reduction in grain boundary density will reduce the number of grain boundary-trapped electrons per unit volume, resulting in the increase of the electron concentration, thus, the electrical conductivity of the film. This explains why the electrical conductivity and electron concentration of the intermediate thick film $(60 \mathrm{~nm})$ are higher than those of the 30-nm-thick film, as shown in Figs. 3(a) and 3(b). When film thickness increases further, the (101) oriented grains are formed on the near surface region and are distributed randomly in the matrix of (100) oriented grains. On the other hand, for intrinsic (undoped) semiconducting oxides charge trapping at grain boundaries is mainly due to dangling bonds and interfacial surface states that depend on the degree of structural ordering at the boundaries. ${ }^{14}$ The structure of the boundaries between (101) and (100) oriented grains is much more disordered than the boundary formed by (100) oriented grains which have three special orientations in the film plane. The increased disordering could introduce a large number of interfacial surface states and increase charge trapped at the grain boundaries. As a result, the conduction electron concentration, and thus the electrical conductivity of the thick film $(100 \mathrm{~nm})$, is lower than that of thinner film $(60 \mathrm{~nm})$. Moreover, the presence of (101) grains can create inhomogeneous conductivity percolation paths that can decrease the overall conductivity of the film. ${ }^{15}$

In conclusion, epitaxial nanocrystalline $\mathrm{SnO}_{2}$ thin films on the (0001) sapphire substrates were fabricated using femtosecond pulsed laser deposition. Detailed analysis using $\mathrm{x}$-ray diffraction and TEM techniques found that the microstructures in terms of grain orientation, size, and shape, depend on the thickness of the films. As a result, the electrical transport properties are strongly dependent on the film thickness.

This work was supported by the National Science Foundation through Grant No. NSF/DMR 9875405 (CAREER, X. Q. P) and by the Petroleum Research Fund (PRF No. 34093G5).

${ }^{1}$ H. L. Tuller, J. Electroceram. 1, 211 (1997).

${ }^{2}$ H. Gleiter, Acta Mater. 1, 48 (2000).

${ }^{3}$ J. Robertson, Phys. Rev. B 30, 3520 (1984).

${ }^{4}$ A. E. Rakhshani, Y. Makdisi, and H. Ramazaniyan, J. Appl. Phys. 83, 1049 (1998)

${ }^{5}$ K. Ihokura and J. Watson, The Stannic Oxide Gas Sensor - Principles and Applications (CRC, Boca Raton, FL, 1994).

${ }^{6}$ G. Advani and A. Jordan, J. Electrochem. Soc. 123, 29 (1990).

${ }^{7}$ C. Xu, J. Tamaki, M. Miur, and N. Yamazoe, Sens. Actuators B 3, 147 (1991).

${ }^{8}$ X. Q. Pan and L. Fu, J. Electroceram. (in press).

${ }^{9}$ X. Q. Pan and L. Fu, J. Appl. Phys. 89, 6048 (2001).

${ }^{10}$ X. Q. Pan, L. Fu, and J. E. Dominguez, J. Appl. Phys. 89, 6056 (2001).

${ }^{11}$ J. Dominguez, L. Fu, X. Q. Pan, P. A. Van Rompay, Z. Y. Zhang, J. A. Nees, and P. P. Pronko, Appl. Phys. Lett. (submitted).

${ }^{12}$ P. A. Mulheran and J. H. Harding, Modell. Simul. Mater. Sci. Eng. 1, 39 (1992).

${ }^{13}$ C. R. M. Grovenor, J. Phys. C 18, 4079 (1985).

${ }^{14}$ M. H. Sukkar and H. L. Tuller, in Surface Chemistry of Oxide Materials, edited by J. Nowotny and L.-C. Dufour (Elseview, Amsterdam, 1988), p. 621.

${ }^{15}$ J. W. Orton and M. J. Powell, Rep. Prog. Phys. 43, 1267 (1980). 